\title{
1 The Analysis of Implied Volatilities
}

\author{
Matthias R. Fengler, Wolfgang Härdle and Peter Schmidt
}

\author{
Institute for Statistics and Econometrics, \\ Department of Business Administration and Economics, \\ Humboldt-Universität zu Berlin, \\ Spandauer Straße 1, 10187 Berlin, Deutschland
}

The analysis of volatility in financial markets has become a first rank issue in modern financial theory and practice: Whether in risk management, portfolio hedging, or option pricing, we need to have a precise notion of the market's expectation of volatility. Much research has been done on the analysis of realized historic volatilities, Roll (1977) and references therein. However, since it seems unsettling to draw conclusions from past to expected market behavior, the focus shifted to implied volatilities, Dumas, Fleming and Whaley (1998). To derive implied volatilities the Black and Scholes (BS) formula is solved for the constant volatility parameter $\sigma$ using observed option prices. This is a more natural approach as the option value is decisively determined by the market's assessment of current and future volatility. Hence implied volatility may be used as an indicator for market expectations over the remaining lifetime of the option.

It is well known that the volatilities implied by observed market prices exhibit a pattern that is far different from the flat constant one used in the BS formula. Instead of finding a constant volatility across strikes, implied volatility appears to be non flat, a stylized fact which has been called "smile" effect. In this chapter we illustrate how implied volatilites can be analyzed. We focus first on a static and visual investigation of implied volatilities, then we concentrate on a dynamic analysis with two variants of principal components and interpret the results in the context of risk management. 


\subsection{Introduction}

Implied volatilities are the focus of interest both in volatility trading and in risk management. As common practice traders directly trade the so called "vega", i.e. the sensitivity of their portfolios with respect to volatility changes. In order to establish vega trades market professionals use delta-gamma neutral hedging strategies which are insensitive to changes in the underlying and to time decay, Taleb (1997). To accomplish this, traders depend on reliable estimates of implied volatilities and - most importantly - their dynamics.

One of the key issues in option risk management is the measurement of the inherent volatility risk, the so called "vega" exposure. Analytically, the "vega" is the first derivative of the BS formula with respect to the volatility parameter $\sigma$, and can be interpreted as a sensitivity of the option value with respect to changes in (implied) volatility. When considering portfolios composed out of a large number of different options, a reduction of the risk factor space can be very useful for assessing the riskiness of the current position. Härdle and Schmidt (2000) outline a procedure for using principal components analysis (PCA) to determine the maximum loss of option portfolios bearing vega exposure. They decompose the term structure of DAX implied volatilities "at the money" (ATM) into orthogonal factors. The maximum loss, which is defined directly in the risk factor space, is then modeled by the first two factors.

Our study on DAX options is organized as follows: First, we show how to derive and to estimate implied volatilities and the implied volatility surface. A data decription follows. In section 1.3.2, we perfom a standard PCA on the covariance matrix of VDAX returns to identify the dominant factor components driving term structure movements of ATM DAX options. Section 1.3.3 introduces a common principal components approach that enables us to model not only ATM term structure movements of implied volatilities but the dynamics of the "smile" as well. 


\subsection{The Implied Volatility Surface}

\subsubsection{Calculating the Implied Volatility}

The BS formula for the price $C_{t}$ of a European call at time $t$ is given by

$$
\begin{aligned}
C_{t} & =S_{t} \Phi\left(d_{1}\right)-K e^{-r \tau} \Phi\left(d_{2}\right) \\
d_{1} & =\frac{\ln \left(S_{t} / K\right)+\left(r+\frac{1}{2} \sigma^{2}\right) \tau}{\sigma \sqrt{\tau}}, \\
d_{2} & =d_{1}-\sigma \sqrt{\tau}
\end{aligned}
$$

where $\Phi$ denotes the cumulative distribution function of a standard normal random variable. $r$ denotes the risk-free interest rate, $S$ the price of the underlying, $\tau=T-t$ the time to maturity and $K$ the strike price. For ATM options the equality $K=S_{t}$ holds.

The only parameter in the Black and Scholes formula that cannot be observed directly is the actual volatility of the underlying price process. However, we may study the volatility which is implied by option prices observed in the markets, the so called implied volatility: implied volatility is defined as the parameter $\hat{\sigma}$ that yields the actually observed market price of a particular option when substituted into the BS formula. The implied volatility of a European put with the same strike and maturity can be deduced from the "put-call parity"

$$
C_{t}-P_{t}=S_{t}-K e^{-r \tau} .
$$

XploRe offers a fast and convenient numerical way to invert the BS formula in order to recover $\hat{\sigma}$ from the market prices of $C_{t}$ or $P_{t}$.

$\mathrm{y}=\operatorname{Impl} \operatorname{Vola}(\mathrm{x}\{, \operatorname{IVmethod}\})$

calculates implied volatilities.

As numerical procedures both a bisectional method and a Newton-Raphson algorithm are available. They are selected by the option IVmethod, which can either be the bisection method (IVmethod="bisect") or the default NewtonRaphson. Within arbitrage bounds on the other input parameters there exists 
a unique solution, since the BS formula is globally concave in $\sigma$. The input vector $\mathrm{x}$ contains the data in an $n \times 6$ dimensional matrix, where the first column contains the underlying asset prices $S$, the second the strikes $K$, the third the interest rates $r$ [on a yearly basis], the fourth maturities $\tau$ [in scale of years], the fifth the observed option prices $C_{t}$ and $P_{t}$. The sixth column contains the type of the option, where 0 abbreviates a put and 1 a call. For example, the command ImplVola $\left(100^{\left.\sim 120 \sim 0.05^{\sim} 0.5^{\sim} 1.94 \sim 1\right)}\right.$ yields the implied volatility of a European call at strike $K=120$ with maturity $\tau$ of half a year, where the interest rate is assumed to be $r=5 \%$, the price of the underlying asset $S=100$ and the option price $C_{t}=1.94$ : the result is $\hat{\sigma}=24.94 \%$. One may verify this result by using XploRe:

opc $=$ BlackScholes $(S, K, r$, sigma, tau, task $)$

which calculates European option prices according to the Black and Scholes model, when no dividend is assumed. The first 5 input parameters follow the notation in this paper, and task specifies whether you desire to know a call price, task $=1$, or a put price, task $=0$. Indeed, for $\sigma=24.94 \%$ we reproduce the assumed option call price of $C_{t}=1.94 . \quad \mathbf{Q}_{\mathrm{XFGiv} 00 . \mathrm{xpl}}$

Now we present a more complex example using option data from the German and Swiss Futures Exchange (EUREX). The data set volsurfdata2 . dat contains the full set of option prices (settlement prices) as observed on January 4th, 1999. The first column contains the settlement price $S$ of the DAX, the second the strike price $K$ of the option, the third the interest rate $r$, the fourth time to maturity $\tau$, the fifth the option prices $C_{t}$ or $P_{t}$ and the last column finally the type of option, either 0, i.e. a put, or 1, i.e. a call. Hence the data set is already in the form as required by the quantlet ImplVola. We may therefore use the following code to calculate the implied volatilities:

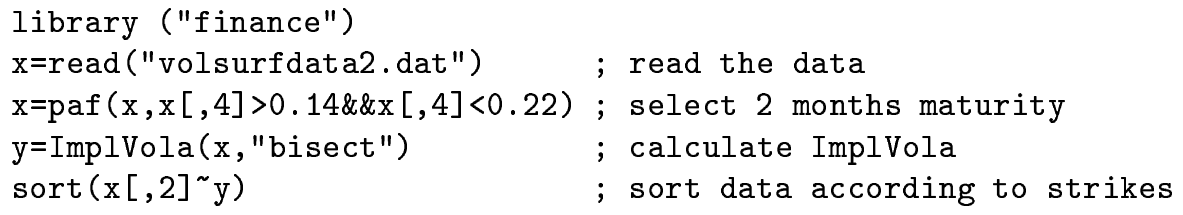




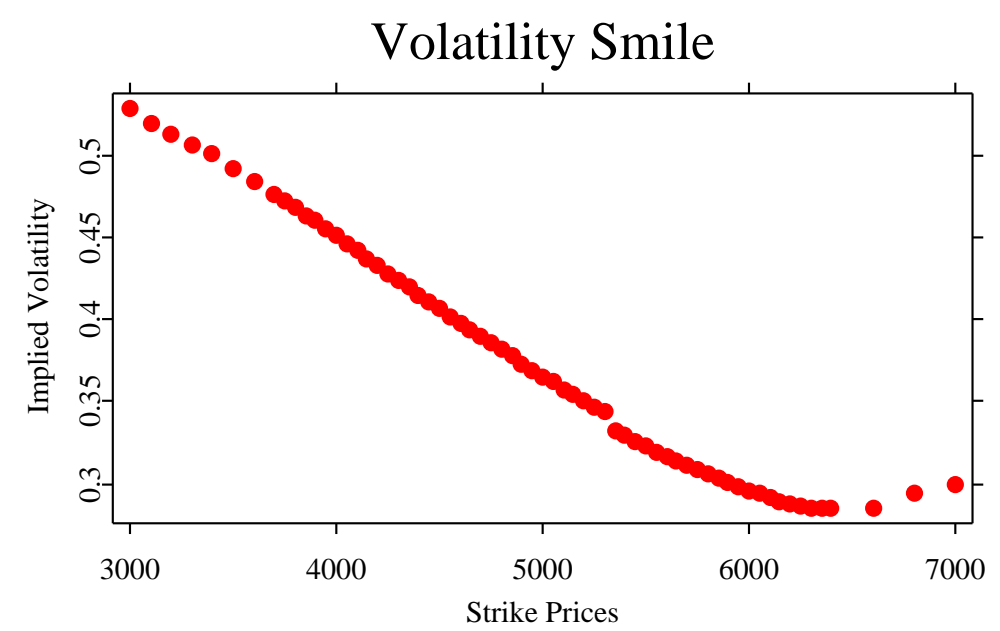

Figure 1.1: Implied volatility "smile" as observed on January 4th, 1999

Q. XFGiv01.xpl

In Figure 1.1 we display the output for the strike dimension. The deviation from the BS model is clearly visible: implied volatilities form a convex "smile" in strikes. One finds a curved shape also across different maturities. In combination with the strike dimension this yields a surface with pronounced curvature (Figure 1.2). The discontinuity of the ATM position is related to tax effects exerting different influences on puts and calls, Hafner and Wallmeier (2001). In our case this effect is not so important, since we smooth the observations and calculate the returns of the implied volatility time series before applying the PCA.

\subsubsection{Surface smoothing}

Calculation of implied volatilities at different strikes and maturities yields a surface. The quantlet volsurf estimates the implied volatility surface on a specified grid using a bi-dimensional kernel smoothing procedure. A NadarayaWatson estimator with a quartic kernel is employed, Aït-Sahalia and Lo (1998), Aït-Sahalia and Lo (2000), Härdle (1990), Härdle, Müller, Sperlich, and Wer- 
watz (2001).

More technically, given a partition of explanatory variables $\left(x_{1}, x_{2}\right)=(K, \tau)$, i.e. of strikes and maturities, the two-dimensional Nadaraya-Watson kernel estimator is

$$
\hat{\sigma}\left(x_{1}, x_{2}\right)=\frac{\sum_{i=1}^{n} K_{1}\left(\frac{x_{1}-x_{1 i}}{h_{1}}\right) K_{2}\left(\frac{x_{2}-x_{2 i}}{h_{2}}\right) \hat{\sigma}_{i}}{\sum_{i=1}^{n} K_{1}\left(\frac{x_{1}-x_{1 i}}{h_{1}}\right) K_{2}\left(\frac{x_{2}-x_{2 i}}{h_{2}}\right)}
$$

where $\hat{\sigma}_{i}$ is the volatility implied by the observed option prices $C_{t i}$ or $P_{t i}$. $K_{1}$ and $K_{2}$ are univariate kernel functions, and $h_{1}$ and $h_{2}$ are bandwidths. The order 2 quartic kernel is given by

$$
K_{i}(u)=\frac{15}{16}\left(1-u^{2}\right)^{2} I(|u| \leq 1) .
$$

The basic structure of volsurf is given by

$\{$ IVsurf, IVpoints $\}=\operatorname{vol} \operatorname{surf}(x$, stepwidth, firstXF, lastXF, firstMat, lastMat, metric, bandwidth, $p$, \{IVmethod\})

As input parameters we first have the $n \times 6$ matrix $\mathrm{x}$ which has been explained in section 1.2.1. The remaining parameters concern the surface: stepwidth is a $2 \times 1$ vector determining the stepwidth in the grid of the surface; the first entry relates to the strike dimension, the second to the dimension across time to maturity. firstXF, lastXF, firstMat, lastMat are scalar constants giving the lowest limit and the highest limit in the strike dimension, and the lowest and the highest limit of time to maturity in the volatility surface. The option metric gives you the choice whether to compute the surface in a moneyness or in a strike metric. Setting metric $=0$ will generate a surface computed in a moneyness metric $K / F$, i.e. strike divided by the (implied) forward price of the underlying, where the forward price is computed by $F_{t}=S_{t} e^{r \tau}$. If metric $=1$, the surface is computed in the original strike dimension in terms of $K$. bandwidth is a $2 \times 1$ vector determining the width of the bins for the kernel estimator. $\mathrm{p}$ determines whether for computation a simple Nadaraya-Watson estimator, $\mathrm{p}=0$, or a local polynomial regression, $\mathrm{p} \neq 0$, is used. The last 
and optional parameter IVmethod has the same meaning as in the ImplVola quantlet. It tells XploRe which method to use for calculating the implied volatilities, default again is Newton-Raphson.

The output are two variables. IVsurf is an $N \times 3$ matrix containing the coordinates of the points computed for the implied volatility surface, where the first column contains the values of the strike dimension, the second those of time to maturity, the third estimated implied volatilities. $N$ is the number of grid points. IVpoints is a $M \times 3$ matrix containing the coordinates of the $M$ options used to estimate the surface. As before, the first column contains the values for the strike dimension, the second the maturity, the third the implied volatilities.

Before presenting an example we briefly introduce a graphical tool for displaying the volatility surface. The following quantlet plots the implied surface:

volsurfplot(IVsurf, IVpoints, \{AdjustToSurface $\}$ )

As input parameters we have the output of volsurf, i.e. the volatility surface IVsurf, and the original observations IVpoints. An optional parameter AdjustToSurface determines whether the surface plot is shown based on the surface data given in IVsurf, or on the basis of the original observations IVpoints. This option might be useful in a situation where you have estimated a smaller part of the surface than would be possible given your data. By default, or AdjustToSurface =1, the graph is adjusted according to the estimated surface.

Now, let's look at an example!

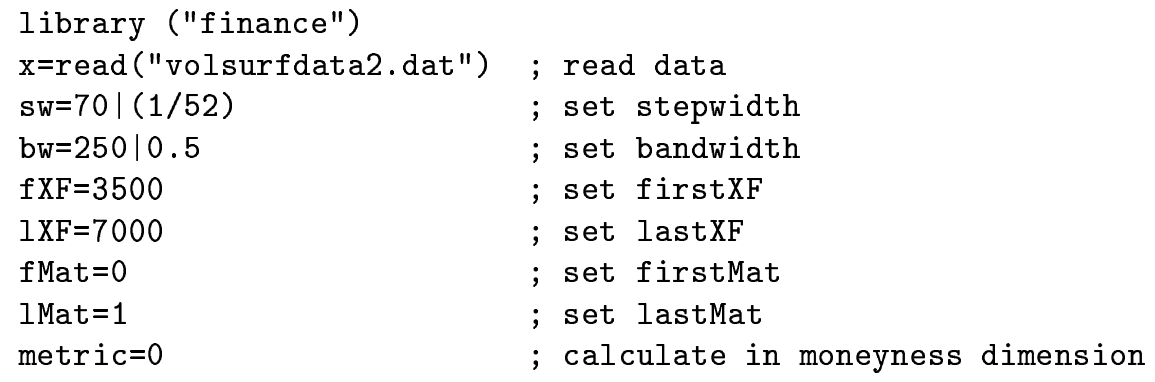


AdjustToSurface $=1$

\{IVSurf, IVpoints\}=volsurf ( $\mathrm{x}, \mathrm{sw}, \mathrm{fXF}, \mathrm{IXF}, \mathrm{fMat}$, lMat, metric, bw, 0)

volsurfplot (IVSurf, IVpoints, AdjustToSurface)

Q. $\mathrm{XFGiv02.xp1}$

XFGiv02 computes an implied volatility surface with the Nadaraya-Watson estimator and displays it (Figure 1.2). The parameters are determined in order to suit the example best, then volsurfplot is used to create the graphic. The output matrix IVsurf contains now all surface values on a grid at the given stepwidth. Doing this for a sequential number of dates produces a time series $\left\{\hat{\sigma}_{t}\right\}$ of implied volatility surfaces. Empirical evidence shows that this surface changes its shape and characteristics as time goes on. This is what we analyze in the subsequent sections.

\subsection{Dynamic Analysis}

\subsubsection{Data description}

Options on the DAX are the most actively traded contracts at the derivatives exchange EUREX. Contracts of various strikes and maturities constitute a liquid market at any specific time. This liquidity yields a rich basket of implied volatilities for many pairs $(K, \tau)$. One subject of our research concerning the dynamics of term structure movements is implied volatility as measured by the German VDAX subindices available from Deutsche Börse AG (http://deutscheboerse.com/)

These indices, representing different option maturities, measure volatility implied in ATM European calls and puts. The VDAX calculations are based on the BS formula. For a detailed discussion on VDAX calculations we refer to Redelberger (1994).

Term structures for ATM DAX options can be derived from VDAX subindices for any given trading day since 18 March 1996. On that day, EUREX started trading in long term options. Shapes of the term structure on subsequent trading days are shown in Figure 1.3.

If we compare the volatility structure of 27 October 1997 (blue line) with that of 28 October 1997 (green line), we easily recognize an overnight upward shift 


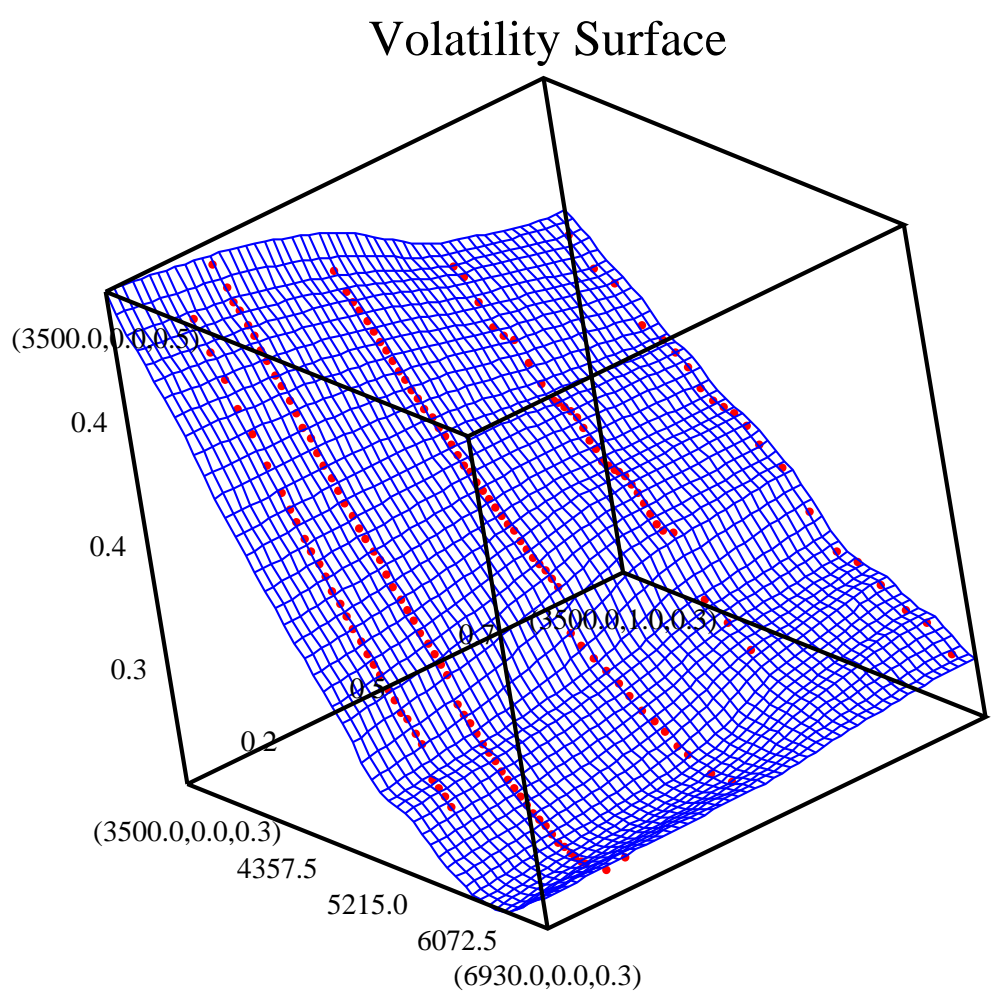

Figure 1.2: Implied volatility surface as observed on January 4th, 1999

Q. XFGiv02.xpl

in the levels of implied volatilities. Moreover, it displays an inversion as short term volatilities are higher than long term ones. Only a couple of weeks later, on 17 November (cyan line) and 20 November (red line), the term structure had normalized at lower levels and showed its typical shape again. Evidently, during the market tumble in fall 1997, the ATM term structure shifted and changed its shape considerably over time.

As an option approaches its expiry date $T$, time to maturity $\tau=T-t$ is declining with each trading day. Hence, in order to analyze the dynamic struc- 


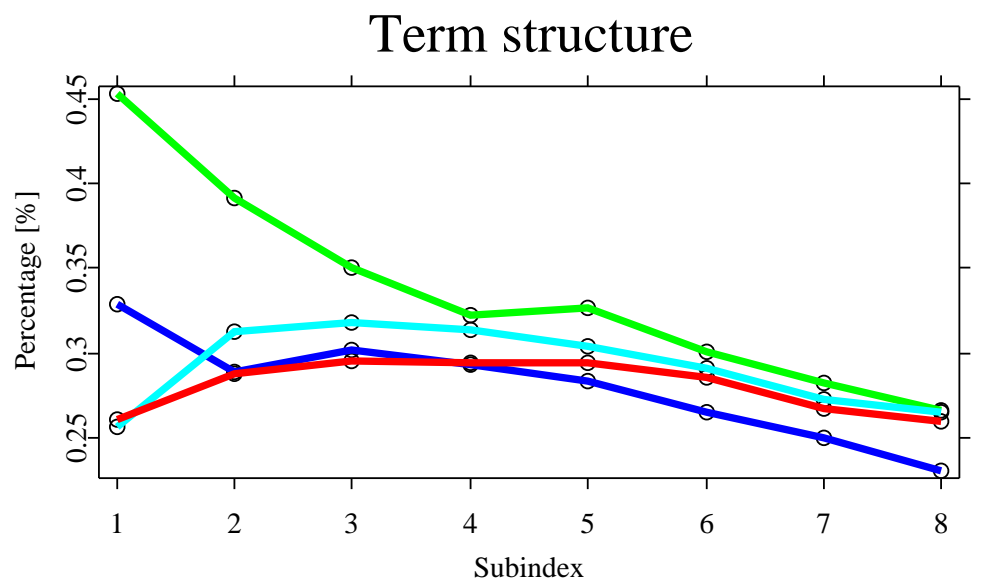

Figure 1.3: Term Structure of VDAX Subindices

Q XFGiv03.xpl

ture of implied volatility surfaces, we need to calibrate $\tau$ as time $t$ passes. To accomplish this calibration we linearly interpolate between neighboring VDAX subindices. For example, to recover the implied volatility $\hat{\sigma}$ at a fixed $\tau$, we used the subindices at $\tau_{-}$and $\tau_{+}$where $\tau_{-} \leq \tau \leq \tau_{+}$, i.e. we computed $\hat{\sigma}_{t}(\tau)$ with fixed maturities of $\tau \in\{30,60,90,180,270,360,540,720\}$ calendar days by

$$
\hat{\sigma}_{t}(\tau)=\hat{\sigma}_{t}\left(\tau_{-}\right)\left[1-\frac{\tau-\tau_{-}}{\tau_{+}-\tau_{-}}\right]+\hat{\sigma}_{t}\left(\tau_{+}\right)\left[\frac{\tau-\tau_{-}}{\tau_{+}-\tau_{-}}\right]
$$

Proceeding this way we obtain 8 time series of fixed maturity. Each time series is a weighted average of two neighboring maturities and contains $n=440$ data points of implied volatilities.

\subsubsection{PCA of ATM Implied Volatilities}

The data set for the analysis of variations of implied volatilities is a collection of term structures as given in Figure 1.3. In order to identify common factors 
we use Principal Components Analysis (PCA). Changes in the term structure can be decomposed by PCA into a set of orthogonal factors.

Define $X_{c}=\left(x_{t j}\right)$ as the $T \times J$ matrix of centered first differences of ATM implied volatilities for subindex $j=1, \ldots, J$ in time $t=1, \ldots, T$, where in our case $J=8$ and $T=440$. The sample covariance matrix $S=T^{-1} X_{c}^{\prime} X_{c}$ can be decomposed by the spectral decomposition into

$$
S=\Gamma \Lambda \Gamma^{T},
$$

where $\Gamma$ is the $8 \times 8$ matrix of eigenvectors and $\Lambda$ the $8 \times 8$ diagonal matrix of eigenvalues $\lambda_{j}$ of $S$. Time series of principal components are obtained by $Y=X_{c} \Gamma$.

A measure of how well the $\mathrm{PCs}$ explain variation of the underlying data is given by the relative proportion $\zeta_{l}$ of the sum of the first $l$ eigenvalues to the overall sum of eigenvalues:

$$
\zeta_{l}=\frac{\sum_{j=1}^{l} \lambda_{j}}{\sum_{j=1}^{8} \lambda_{j}}=\frac{\sum_{j=1}^{l} \operatorname{Var}\left(y_{j}\right)}{\sum_{j=1}^{8} \operatorname{Var}\left(y_{j}\right)} \quad \text { for } \quad l<8
$$

The quantlet XFGiv04 uses the VDAX data to estimate the proportion of variance $\zeta_{l}$ explained by the first $l$ PCs.

Q XFGiv04.xpl

As the result shows the first PC captures around $70 \%$ of the total data variability. The second PC captures an additional $13 \%$. The third PC explains a considerably smaller amount of total variation. Thus, the two dominant PCs together explain around $83 \%$ of the total variance in implied ATM volatilities for DAX options. Taking only the first two factors, i.e. those capturing around $83 \%$ in the data, the time series of implied ATM volatilities can therefore be represented by a factor model of reduced dimension:

$$
x_{t j}=\gamma_{j 1} y_{t 1}+\gamma_{j 2} y_{t 2}+\epsilon_{t},
$$

where $\gamma_{j k}$ denotes the $j k$ th element of $\Gamma=\left(\gamma_{j k}\right), y_{t k}$ is taken from the matrix of principal components $Y$, and $\epsilon_{t}$ denotes white noise. The $\gamma_{j}$ are in fact the sensitivities of the implied volatility time series to shocks on the principal 
components. As is evident from Figure 1.4, a shock on the first factor tends to affect all maturities in a similar manner, causing a non-parallel shift of the term structure. A shock in the second factor has a strong negative impact on the front maturity but a positive impact on the longer ones, thus causing a change of curvature in the term structure of implied volatilities.

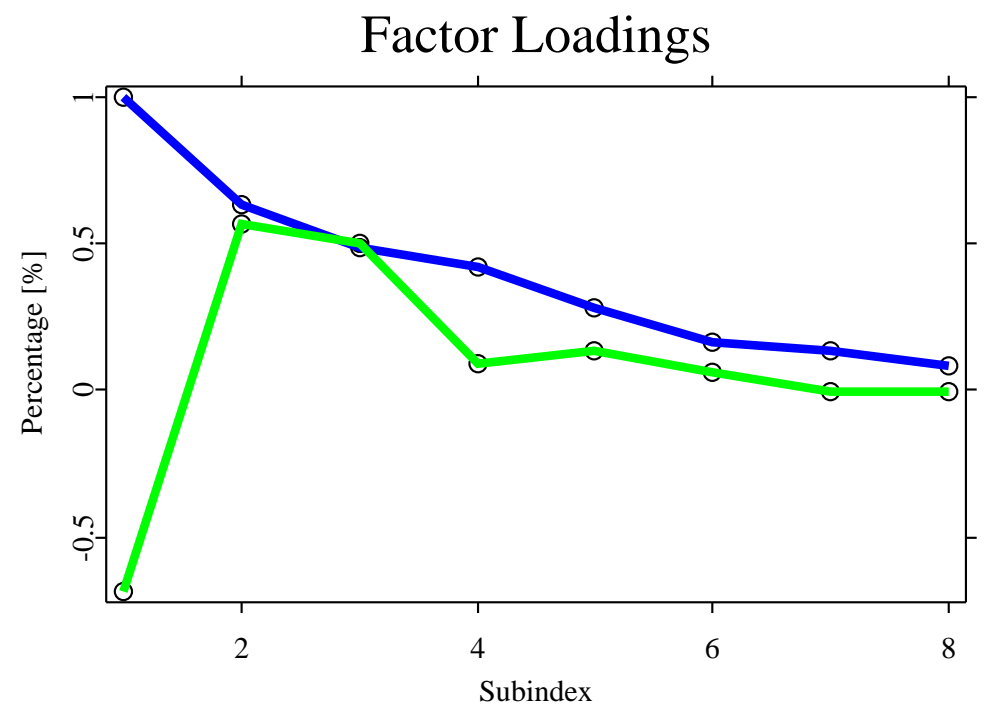

Figure 1.4: Factor Loadings of First and Second PC

Q XFGiv05.xpl

\subsubsection{Common PCA of the Implied Volatility Surface}

Implied volatilities calculated for different strikes and maturities constitute a surface. The principle component analysis as outlined above, does not take this structure into account, since only one slice of the surface, the term structure of ATM options are used. In this section we present a technique that allows us to analyze several slices of the surface simultaneously. Since options naturally fall into maturity groups, one could analyze several slices of the surface taken at different maturities. What we propose to do is a principal component analysis 
of these different groups. Enlarging the basis of analysis will lead to a better understanding of the dynamics of the surface. Moreover, from a statistical point of view, estimating PCs simultaneously in different groups will result in a joint dimension reducing transformation. In this chapter, we like to present a form of a multi-group PCA, the so called common principle components analysis (CPCA), which yields this joint eigenstructure across groups.

In addition to traditional PCA, the basic assumption of CPCA is that the space spanned by the eigenvectors is identical across several groups, whereas variances associated with the components are allowed to vary. This approach permits us to analyze a $p$ variate random vector in $k$ groups, say $k$ maturities of implied volatilities jointly, Fengler, Härdle and Villa (2001).

More formally, the hypothesis of common principle components can be stated in the following way, Flury (1988):

$$
H_{C P C}: \Psi_{i}=\Gamma \Lambda_{i} \Gamma^{T}, \quad i=1, \ldots, k
$$

where the $\Psi_{i}$ are positive definite $p \times p$ population covariance matrices, $\Gamma=$ $\left(\gamma_{1}, \ldots, \gamma_{p}\right)$ is an orthogonal $p \times p$ transformation matrix and $\Lambda_{i}=\operatorname{diag}\left(\lambda_{i 1}, \ldots, \lambda_{i p}\right)$ is the matrix of eigenvalues. Moreover, assume that all $\lambda_{i}$ are distinct.

Let $S$ be the (unbiased) sample covariance matrix of an underlying $p$-variate normal distribution $N_{p}(\mu, \Psi)$ with sample size $n$. Then the distribution of $n S$ is Wishart, Muirhead (1982), p. 86, with $n-1$ degrees of freedom:

$$
n S \sim \mathcal{W}_{p}(\Psi, n-1)
$$

The density of the Wishart distribution is given by

$$
\begin{gathered}
f(S)=\frac{1}{\Gamma_{p}\left(\frac{n-1}{2}\right)|\Psi|^{(n-1) / 2}}\left(\frac{n-1}{2}\right)^{\frac{p(n-1)}{2}} \times \\
\quad \exp \left\{\operatorname{tr}\left(-\frac{n-1}{2} \Psi^{-1} S\right)\right\}|S|^{(n-p-2) / 2},
\end{gathered}
$$

where

$$
\Gamma_{p}(x)=\pi^{p(p-1) / 4} \prod_{i=1}^{p} \Gamma\left\{x-\frac{1}{2}(i-1)\right\}
$$


is the multivariate gamma function, Muirhead (1982). Hence for given Wishart matrices $S_{i}$ with sample size $n_{i}$ the likelihood function can be written as

$$
L\left(\Psi_{1}, \ldots, \Psi_{k}\right)=C \prod_{i=1}^{k} \exp \left\{\operatorname{tr}\left(-\frac{1}{2}\left(n_{i}-1\right) \Psi_{i}^{-1} S_{i}\right)\right\}\left|\Psi_{i}\right|^{-\frac{1}{2}\left(n_{i}-1\right)}
$$

where $C$ is a constant not depending on the parameters $\Psi_{i}$. Maximizing the likelihood is equivalent to minimizing the function

$$
g\left(\Psi_{1}, \ldots, \Psi_{k}\right)=\sum_{i=1}^{k}\left(n_{i}-1\right)\left\{\ln \left|\Psi_{i}\right|+\operatorname{tr}\left(\Psi_{i}^{-1} S_{i}\right)\right\} .
$$

Assuming that $H_{C P C}$ holds, i.e. in replacing $\Psi_{i}$ by $\Gamma \Lambda_{i} \Gamma^{T}$, one gets after some manipulations

$$
g\left(\Gamma, \Lambda_{1}, \ldots, \Lambda_{k}\right)=\sum_{i=1}^{k}\left(n_{i}-1\right) \sum_{j=1}^{p}\left(\ln \lambda_{i j}+\frac{\gamma_{j}^{T} S_{i} \gamma_{j}}{\lambda_{i j}}\right) .
$$

As we know from section 1.3.2, the vectors $\gamma_{j}$ in $\Gamma$ need to be orthogonal. We achieve orthogonality of the vectors $\gamma_{j}$ via the Lagrange method, i.e. we impose the $p$ constraints $\gamma_{j}^{T} \gamma_{j}=1$ using the Lagrange multiplyers $\mu_{j}$, and the remaining $p(p-1) / 2$ constraints $\gamma_{h}^{T} \gamma_{j}=0$ for $(h \neq j)$ using the multiplyer $\mu_{h j}$. This yields

$$
g^{*}\left(\Gamma, \Lambda_{1}, \ldots, \Lambda_{k}\right)=g(\cdot)-\sum_{j=1}^{p} \mu_{j}\left(\gamma_{j}^{T} \gamma_{j}-1\right)-2 \sum_{h<j}^{p} \mu_{h j} \gamma_{h}^{T} \gamma_{j} .
$$

Taking partial derivatives with respect to all $\lambda_{i m}$ and $\gamma_{m}$, it can be shown (Flury, 1988) that the solution of the CPC model is given by the generalized system of characteristic equations

$$
\gamma_{m}^{T}\left(\sum_{i=1}^{k}\left(n_{i}-1\right) \frac{\lambda_{i m}-\lambda_{i j}}{\lambda_{i m} \lambda_{i j}} S_{i}\right) \gamma_{j}=0, \quad m, j=1, \ldots, p, \quad m \neq j
$$


This has to be solved using

$$
\lambda_{i m}=\gamma_{m}^{T} S \gamma_{m}, \quad i=1, \ldots, k, \quad m=1, \ldots, p
$$

under the constraints

$$
\gamma_{m}^{T} \gamma_{j}=\left\{\begin{array}{ll}
0 & m \neq j \\
1 & m=j
\end{array} .\right.
$$

Flury (1988) proves existence and uniqueness of the maximum of the likelihood function, and Flury and Gautschi (1988) provide a numerical algorithm, which has been implemented in the quantlet CPC.

\section{CPC-Analysis}

A number of quantlets are designed for an analysis of covariance matrices, amongst them the CPC quantlet:

$\{B$, betaerror, lambda, lambdaerror, psi $\}=\operatorname{CPC}(\mathrm{A}, \mathrm{N})$

estimates a common principle components model.

As input variables we need a $p \times p \times k$ array A, produced from $k p \times p$ covariance matrices, and a $k \times 1$ vector of weights $N$. Weights are the number of observations in each of the $k$ groups.

The quantlet produces the $p \times p$ common transformation matrix $\mathrm{B}$, and the $p \times p$ matrix of asymptotic standard errors betaerror. Next, eigenvalues lambda and corresponding standard errors lamdbaerror are given in a vector array of $1 \times p \times k$. Estimated population covariances psi are also provided. As an example we provide the data sets volsurf01.dat, volsurf02.dat and volsurf03.dat that have been used in Fengler, Härdle and Villa (2001) to estimate common principle components for the implied volatility surfaces of the DAX 1999. The data has been generated by smoothing a surface day by day as spelled out in section 1.2.2 on a specified grid. Next, the estimated grid points have been grouped into maturities of $\tau=1, \tau=2$ and $\tau=3$ months 
and transformed into a vector of time series of the "smile", i.e. each element of the vector belongs to a distinct moneyness ranging from 0.85 to 1.10.

The following quantlet shows how to estimate a CPC model: $\mathbf{Q}$ XFGiv06.xpl

We plot the first three eigenvectors in a parallel coordinate plot in Figure 1.5. The basic structure of the first three eigenvectors is not altered. We find a shift, a slope and a twist structure. This structure is common to all maturity groups, i.e. when exploiting PCA as a dimension reducing tool, the same transformation applies to each group! However, from comparing the size of eigenvalues among groups, i.e. ZZ. Iambda, we find that variability is dropping across groups as we move from the front contracts to long term contracts.

\section{Common Coordinate Plot: First three Eigenvectors}

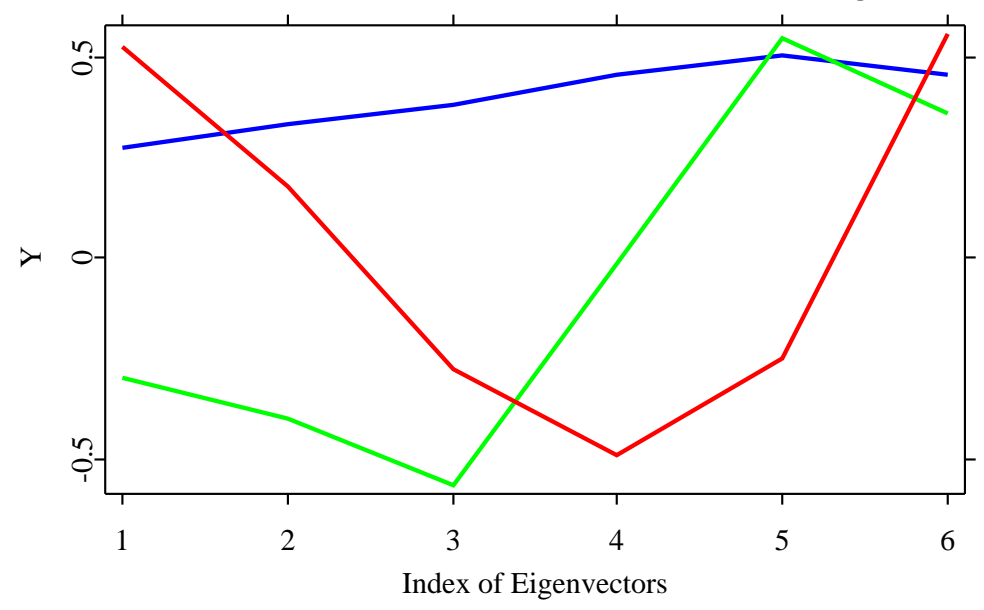

Figure 1.5: Factor loadings of the first (blue), the second (green), and the third PC (red)

Q. XFGiv06.xpl

Before drawing conclusions we should convince ourselves that the CPC model is truly a good description of the data. This can be done by using a likelihood ratio test. The likelihood ratio statistic for comparing a restricted (the CPC) model against the unrestricted model (the model where all covariances are 
treated separately) is given by

$$
T_{\left(n_{1}, n_{2}, \ldots, n_{k}\right)}=-2 \ln \frac{L\left(\widehat{\Psi}_{1}, \ldots, \widehat{\Psi}_{k}\right)}{L\left(S_{1}, \ldots, S_{k}\right)} .
$$

Inserting from the likelihood function we find that this is equivalent to

$$
T_{\left(n_{1}, n_{2}, \ldots, n_{k}\right)}=\sum_{i=1}^{k}\left(n_{i}-1\right) \frac{\operatorname{det} \widehat{\Psi}_{i}}{\operatorname{det} S_{i}},
$$

which is $\chi^{2}$ distributed as $\min \left(n_{i}\right)$ tends to infinity with

$$
k\left\{\frac{1}{2} p(p-1)+1\right\}-\left\{\frac{1}{2} p(p-1)+k p\right\}=\frac{1}{2}(k-1) p(p-1)
$$

degrees of freedom. In the quantlet XFGiv06 this test is also included.

Q XFGiv06.xpl

The calculations yield $T_{\left(n_{1}, n_{2}, \ldots, n_{k}\right)}=31.836$, which corresponds to the $p$-value $p=0.37512$ for the $\chi^{2}(30)$ distribution. Hence we cannot reject the CPC model against the unrelated model, where PCA is applied to each maturity separately.

In following section 1.3.2, we can estimate the amount of variability $\zeta_{l}$ explained by the first $l$ principle components: again a few number of factors, up to three at the most, is capable of capturing a large amount of total variability present in the data. Since the model now captures variability both in strike and maturity dimension, this can be a suitable starting point for a simplified VaR calculation for delta-gamma neutral option portfolios using Monte Carlo methods, and is hence a valuable insight for risk management. 


\section{Bibliography}

Aït-Sahalia, Y. and Lo, A. W. (1998). Nonparametric Estimation of State-Price Densities Implicit in Financial Assets, Journal of Finance Vol. LIII, 2, pp. $499-547$.

Aït-Sahalia, Y. and Lo, A. W. (2000). Nonparametric Risk management and implied risk aversion, Journal of Econometrics 94, pp. 9-51.

Dumas, B., Fleming, J. and Whaley, R. E. (1998). Implied Volatility Functions: Empirical Tests, Journal of Finance Vol. LIII, 6, pp. 2059-2106.

Fengler, M. R., Härdle, W. and Villa, Chr. (2001). The Dynamics of Implied Volatilities: A Common Principal Components Approach, SfB 373 Discussion Paper No. 2001/38, HU Berlin.

Flury, B. (1988). Common Principle Components Analysis and Related Multivariate Models, Wiley Series in Probability and Mathematical Statistics, John Wiley \& Sons, New York.

Flury, B. and Gautschi, W. (1986). An Algorithm for simultaneous orthogonal transformation of several positive definite symmetric matrices to nearly diagonal form SIAM Journal on Scientific and Statistical Computing,7, pp. $169-184$.

Härdle, W.(1990). Applied Nonparametric Regression, Econometric Society Monographs 19, Cambridge University Press.

Härdle, W., Müller, M., Sperlich, S. and Werwartz, A. (2001). Non - and Semiparametric Modelling, Springer e-book, http://www.xplorestat.de/ebooks/ebooks.html.

Härdle, W. and Schmidt, P. (2000). Common Factors Governing VDAX Movements and the Maximum Loss, SFB 373 Discussion Paper, No.: 97/00, HU Berlin. 
Hafner, R. and Wallmeier, M. (2001). The Dynamics of DAX Implied Volatilities, International Quarterly Journal of Finance,1, 1, pp. 1-27.

Muirhead, R. J. (1982). Aspects of Multivariate Statistics, Wiley Series in Probability and Mathematical Statistics, John Wiley \& Sons, New York.

Redelberger, T. (1994). Grundlagen und Konstruktion des VDAXVolatilitätsindex der Deutsche Börse AG, Deutsche Börse AG, Frankfurt am Main.

Roll, R. (1977). A Critique of the Asset Pricing Theory's Tests: Part I, Journal of Financial Economics,4, pp. 129-176.

Taleb, N. (1997). Dynamic Hedging: Managing Vanilla and Exotic Options, John Wiley \& Sons, New York.

Villa, C. and Sylla, A. (2000). Measuring implied surface risk using PCA in Franke, J., Härdle, W. and Stahl, G.: Measuring Risk in Complex Stochastic Systems, LNS 147, Springer Verlag, New York, pp. 131-147. 